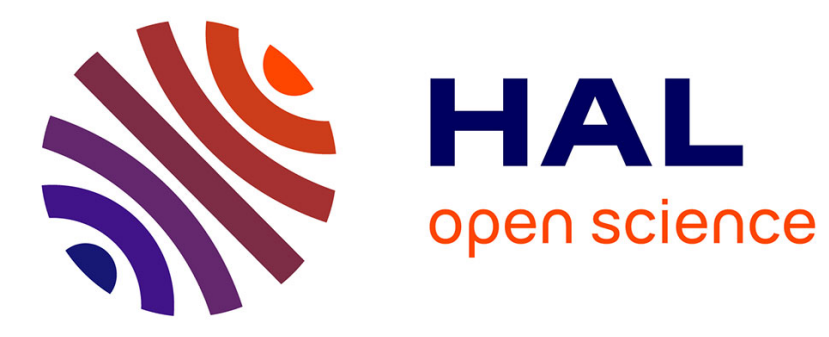

\title{
Dynamics of long-term statin therapy
}

Maarit Jaana Korhonen, Arja Helin-Salmivaara, Risto Huupponen

\section{To cite this version:}

Maarit Jaana Korhonen, Arja Helin-Salmivaara, Risto Huupponen. Dynamics of long-term statin therapy. European Journal of Clinical Pharmacology, 2011, 67 (9), pp.925-931. 10.1007/s00228-0111019-2 . hal-00679600

\section{HAL Id: hal-00679600 https://hal.science/hal-00679600}

Submitted on 16 Mar 2012

HAL is a multi-disciplinary open access archive for the deposit and dissemination of scientific research documents, whether they are published or not. The documents may come from teaching and research institutions in France or abroad, or from public or private research centers.
L'archive ouverte pluridisciplinaire HAL, est destinée au dépôt et à la diffusion de documents scientifiques de niveau recherche, publiés ou non, émanant des établissements d'enseignement et de recherche français ou étrangers, des laboratoires publics ou privés. 
Maarit Jaana Korhonen, PhD, LicSci (Pharm) ${ }^{1,2}$; Arja Helin-Salmivaara, PhD, MD ${ }^{1,3}$

Risto Huupponen, $\mathrm{PhD}, \mathrm{MD}^{1,4}$

\section{Dynamics of Long-Term Statin Therapy}

${ }^{1}$ Department of Pharmacology, Drug Development and Therapeutics, University of Turku, Finland

${ }^{2}$ Institute of Public Health and Clinical Nutrition, Faculty of Health Sciences, University of Eastern

Finland, Kuopio, Finland

3 Unit of General Practice, Hospital District of Helsinki and Uusimaa, Helsinki, Finland

${ }^{4}$ Unit of Clinical Pharmacology, Turku University Hospital, Turku, Finland

Corresponding author:

Maarit Jaana Korhonen

Department of Pharmacology, Drug Development and Therapeutics, University of Turku, 20014 Turun yliopisto, Finland

Tel. +358403572081

Fax. +35817162424

E-mail: maarit.korhonen@utu.fi 


\section{Abstract (250 words)}

Purpose Knowledge of different usage patterns emerging during a long-term statin therapy is limited. We aimed at characterizing statin use, including rates of reinitiation after extended periods of non-use, transitions between good and poor adherence, and the effect of the length of a drug-free period on identification of new use.

Methods Persons aged 45-84 purchasing statins in 1997 and 2007 were identified through the Finnish prescription register. New users in 1997, including those with no statin purchases during the preceding 3 years, were followed-up until institutionalization, death or Dec 31, 2007. Reinitiation rates after $\geq 180$ days with no refill for statins were analyzed using survival analysis. Annual adherence levels and the prevalences of good (proportion of days covered $\geq 0.80)$ and poor $(<0.80)$ adherence were calculated for 10 one-year periods since initiation. For statin users in 2007, purchases were captured over the previous 10 years.

Results 32,760 persons initiated statin treatment in $1997,48.1 \%$ of whom discontinued it for at least 180 days by the end of follow-up. Of the discontinuers, $46.7 \%$ restarted the treatment within 1 year and $88.7 \%$ by the end of follow-up. Of those followed-up for 10 years or longer, $51.8 \%$ accumulated 6 or more years of treatment with good adherence. In 2007, 27.7\% of the initiators having no statin purchases within the previous year had refills during the preceding 10 years.

Conclusion Statin use is dynamic. This should be taken into consideration in clinical practice and when studying the incidence, patterns and health outcomes of statin use.

Key words adherence, drug use pattern, claims data, misclassification 


\section{Introduction}

Statins have been shown to reduce the risk of cardiovascular events in a wide variety of high-risk patients [1-2]; however, benefits of statin therapy may be compromised because of suboptimal persistence with and adherence to it [3-5]. One quarter to $50 \%$ of new statin users discontinue the therapy during the first year [6-9]. In Finland, 44\% of persons initiating statin use in 1995 continued it for 10 years or longer [9]. The pattern of statin use remained stable in 1995-2004, with a slight improvement in persistence rates for the initiators in 1998-2004 [10]. Information on discontinuation rates is important also because the interruption of statin use may exert an excess risk of cardiovascular events and death in some patients groups [11-12], potentially attributable to a biologic rebound phenomenon [13]. However, studies ending the follow-up at the first extended break in refills are likely to underestimate the patient's cumulative statin exposure. Actually, the studies reporting on reinitation rates indicate that the majority of the stoppers resume statin use later on in their life [14-15].

Observational studies on statin effectiveness typically employ new-user designs, restricting a study cohort to initiators. The new-user design allows for estimation of the effects associated with drug initiation and adjustment for confounding due to predictors of persistence with drug therapy, and also avoids overadjustment for potential consequences of drug treatment [16]. Nevertheless, no consensus exists on the required time period without statin use before the first observed use. In administrative database studies, periods without any statin (or any lipid-lowering drug [4]) dispensations prior to the cohort entry have ranged from 3-6 months [17-18] to $1-3$ years [3-5, 19-20]. The choice of this so called washout period is likely to be motivated by the reimbursement, prescribing and dispensing rules of the healthcare system through which the data for a specific study are generated. Especially when studying refill patterns, it may be reasonable to select the washout period according to the characteristics of the data source; however, differences in washout periods across studies may hamper the comparison of the risk 
estimates of health outcomes obtained in them.

The aim of this study was two-fold: to characterize the patterns of long-term statin use, including the rates of reinitiation after extended periods of non-use and transitions between good and poor adherence, and to describe the effect of the length of drug-free period on identification of new use, in samples of statin initiators in Finland.

\section{Methods}

\section{Reimbursement for and pricing of statins in Finland}

In Finland, statins are available on prescription only. The National Health Insurance Scheme, run by the Social Insurance Institution of Finland (SII), provides prescription drug coverage for all about 5 million community-dwelling residents of the country [21]. The drug reimbursement system consists of three refund categories (basic, lower special and higher special refund) graded according to medical criteria based on the severity of the illness and the necessity of the drug therapy [22]. Statins are reimbursed under the basic refund category except for the patients entitled to lower special refund due to dyslipidemia associated with coronary artery disease (CAD) (since 2000) or familial hypercholesterolemia. The copayment for the basic refund category was 10 euros per purchase and 50\% of the balance of the remaining price until the end of 2005. For the lower special refund category, the respective figures were 5 euros and $25 \%$. Thereafter, $42 \%$ and $72 \%$ of the price of each drug has been reimbursed. Generic simvastatin has been available since 2003 after which the prices of simvastatin products decreased dramatically. Beginning October 2006, the prescribing of less expensive statins was promoted by a restriction of the reimbursement of the most expensive ones, atorvastatin and rosuvastatin. These two statins remained reimbursable only for treatment of severe disorders of lipid metabolism in 
high risk patients who were not able to use less expensive statins due to intolerance or ineffectiveness [23]. In practice, users of atorvastatin and rosuvastatin were entitled to reimbursement only after they had tried less expensive statins first.

\section{Data sources}

Since 1994, the SII has maintained an electronic pharmacy claims database called the Prescription Register. The Register contains records of all drug purchases reimbursed to residents in non-institutional settings [21]. For each drug, dispensing date, the WHO Anatomical Therapeutic Chemical (ATC) classification code [24], strength, and quantity dispensed are recorded. For each person, information on birth and death dates and gender is available. Persons institutionalized permanently, including those staying in public nursing homes and hospitals for $>90$ days without interruption, are not eligible for reimbursements, and their purchases are not registered. These persons can be identified from a separate SII register.

Because of the reliance on the above administrative data, the expression statin use in this report refers to purchasing statins or being dispensed statin prescriptions in a pharmacy. The actual use of statins could not be verified.

Study samples

There were two separate samples. The first study sample consisted of persons initiating statin therapy (ATC code C10AA) during the year 1997. We defined an initiator (new statin user) as a person being dispensed a statin prescription without having been dispensed one in the preceding 3 years (1095 days). As a sensitivity analysis, the above definition was relaxed so that all persons with no dispensations for 
statins during the preceding year (365 days) were considered as initiators. We followed the initiators up until they died, were institutionalized permanently according to the SII register, or reached the end of follow-up or Dec 31, 2007, whichever came first.

For identifying discontinuation dates, we created a drug coverage data file for each initiator. We linked consecutive prescriptions together based on dispending dates and the number of days' supply of each prescription, assuming a dosage of one tablet per day. If a prescription was filled when there should have been unused tablets from the previous dispensation, we assumed use of the new supply to begin after the end of the previous supply. We defined discontinuation as failing to fill a prescription within 180 days of finishing the previous one. In sensitivity analyses, this gap was defined as 90 and 270 days. According to the Finnish drug reimbursement scheme, a maximum of 3 months' supply of medication can be reimbursed at one transaction; therefore, gaps applicable in other reimbursement systems may be too short in the Finnish context. We analyzed statins as a group, considering switching between various statins as continuation of the therapy.

We operationalized adherence as the proportion of days covered (PDC) [25]. Based on reimbursed statin purchases and assuming a dosage of one tablet per day, we obtained the PDC with a statin for each year (365-day period) of follow-up by dividing the number of days on which the person had a statin on-hand by 365 . Good adherence refers to $\mathrm{PDC} \geq 0.80[6,26]$.

The second sample included all persons who had purchased statins in 2007. For this sample, we defined new statin users by varying the requirement for the length of the period without statin dispensations prior to the first prescription in 2007 from 3 months [17] up to 9 years. We calculated percentages of persons misclassified in each case, defining the persons with no statin dispensations during the previous 10 or more years as true new users. 


\section{Statistical analysis}

For comparing characteristics of those qualifying and not qualifying as statin initiators when employing different purchase-free gaps, we used the $\chi^{2}$ square test and the t-test. For the discontinuers in the 1997 sample, we estimated the distribution of the time until a person refilled a statin prescription with the Kaplan-Meier method, using SAS software (version 9.1, SAS Institute Inc., Cary, NC). There was no legal requirement for approval by an ethics committee since we used de-identified data and did not contact the persons.

\section{Results}

Characteristics of the initiators in 1997

A cohort of 32,760 statin initiators, i.e. those with no statin purchases during the preceding 3 years, aged 45-84 in 1997 was identified. The initiators had a mean age of 63 years; slightly less than half were male (Table 1). As identified in the SII Special Reimbursement Register, 10,682 (32.6\%) had an established CAD and $88(0.3 \%)$ had familial hypercholesterolemia. The initial statin was most commonly simvastatin (Table 1). The mean follow-up time was 8.2 years (SD, 3.4), 10,357 (31.6\%) being censored because of institutionalization and $2825(8.6 \%)$ because of death.

In sensitivity analyses, we relaxed the definition for new users to include all those with no statin dispensations during the preceding 1-year period: the number of 45-84 year-old initiators identified this way was 36,581 (Table 1). Compared with those qualifying as initiators with the 3 -year gap (Table 1), the 3821 persons qualifying with the 1-year but not with the 3-year gap were more commonly male (55.5\%) and somewhat younger (mean age, 62.3 years; $\mathrm{SD}, 8.5$ years). In addition, they initiated their statin therapy more commonly with lovastatin (23.7\%) and less commonly with simvastatin $(40.8 \%)$ or 
fluvastatin $(29.0 \%$, p-values for all comparisons $<0.0001)$.

Patterns of statin use in the 1997 cohort

Of the original cohort $(n=32,760), 15,759$ or almost every second $(48.1 \%)$ had at least one statin-free period lasting for 180 days or longer during the follow-up. The number of initiators with at least one 90 day statin-free gap was 20,840 (63.6\%) and the number of those with at least a 270-day gap was 13,165 (40.2\%), respectively. Of those with 90 -, 180 - and 270 -day gaps, estimated $62.4 \%, 46.7 \%$ and $36.7 \%$ reinitiated statin therapy within 1 year after the end of the first gap (Fig 1). By the end of follow-up, the reinitiation rates were $92.3 \%, 88.7 \%$ and $86.2 \%$, respectively.

Of those surviving at least 365 days since statin initiation $(n=30,893), 14,886(48.2 \%)$ had good first-year adherence ( $\mathrm{PDC} \geq 0.80$, Table 2) while 2874 (9.3\%) purchased only one statin prescription by the end of the first year. The proportion of those with good adherence increased up to $58.8 \%$ by the tenth year of follow-up. The proportion of those with continuous good adherence throughout the 10 years (17.1\%) was the same as the proportion of those never achieving good adherence in any of the 10 years (17.3\%) (Fig 2). Altogether $51.8 \%$ of those followed up for 10 years used statins with good adherence for a total of 6 years or longer, and $69.2 \%$ for 3 years or longer (total number of 1-year gaps in good adherence at most 4 and 7 , respectively).

Sensitivity analyses revealed similar patterns of discontinuation, reinitiation and adherence in the cohort identified using the relaxed definition of new use $(n=36,518)$. The proportions of initiators with at least one statin-free period lasting 90, 180, and 270 days during the follow-up were $64.7 \%, 49.2 \%$ and $41.1 \%$. Estimated $62.8 \%, 47.7 \%$ and $38.2 \%$ of those with $90-$, 180 - and 270 -day gaps restarted the therapy within 1 year after the end of the first gap. By the end of follow-up, the estimated probabilities of reinitiation were $92.5 \%, 89.1 \%$ and $86.8 \%$, respectively. Of those surviving at least one year since initiation 
$(n=34,517), 47.2 \%$ had good adherence and 9.7\% purchased only one prescription during that year. One half $(51.1 \%)$ of those followed up 10 or more years after initiation $(n=22,486)$ accumulated at least 6 oneyear periods of good adherence.

Characteristics and previous statin purchases of the initiators in 2007

In total, 520,894 Finns aged 45-84 purchased statins in 2007. Of the 78,512 new statin users defined as those who had not purchased statins during the preceding 3 years, $89.3 \%$ initiated with simvastatin (Table 1). Every fifth (21.0\%) of the 99,348 initiators defined as those with a 1-year purchase-free period did not qualify as such when using a 3-year gap. These 20,836 initiators differed from those with no dispensations during the previous 3 years (Table 1) being older (mean age, 64.4; SD, 9.7 years) and less commonly male (49.2\%). Among them the initial statin was less commonly simvastatin $(70.4 \%)$ and more commonly atorvastatin $(11.0 \%)$ rosuvastatin $(5.6 \%)$, fluvastatin $(5.7 \%)$, pravastatin $(4.1 \%)$, lovastatin $(2.0 \%)$ or a combination of simvastain and ezetimibe $(1.2 \%)$ (p-values for all comparisons $<0.0001)$

Overall, the number of new users decreased rapidly with the increasing length of statin-free period required before the first statin purchase in 2007: $27.7 \%$ of the new users defined as those with a 1-year purchase-free period did not qualify as such when using a 10-year gap (Fig 3). When using a 1-year gap, women aged 65-84 were somewhat more commonly (34.1\%) misclassified as new users than were men of the same age (30.1\%) or women and men aged $45-64(24.1 \%$ and $24.8 \%)$.

\section{Discussion}

Our observations based on unselected populations of statin initiators from a country with universal prescription drug coverage support the view that long-term statin use is dynamic [15]. More specifically, 
we observed that most statin users had at least 1 extended period of non-use during an average of 8.2 years of follow-up. The majority (86-92\%) of those who stopped statin use, however, reinitiated it. Accordingly, almost $30 \%$ of persons with no statin purchases within 1 year preceding the initation (a conventional definition for new use) were not statin-naíve when the required period of no statin purchases was extended to the previous 10 years. Finally, while only every sixth statin initiator maintained good adherence and was therefore supposedly exposed to an optimal statin therapy throughout the whole 10year period, every second accumulated altogether 6 or more years of such exposure.

Incidence of 90- and 180-day treatment gaps and also the rates of reinitiation after the first gap were slightly higher in our study than in a Canadian study observing persons who initiated statin therapy between Jan 1997 and June 2004 [15]. This may partly be explained by the differences in reimbursement rules affecting the refill patterns, the typical duration of a statin prescription being 1 month in Canada and 3 months in Finland. Indeed, our sensitivity analyses showed that the estimated rates of discontinuation and reinitiation depend considerably on the gap length chosen, supporting our view that applying shorter gap lengths may overestimate the rate of interruptions in statin use in the Finnish context.

Interestingly, our estimates on discontinuation and restarting of and good adherence to statin therapy were only marginally affected by the definition of new use in 1997. However, while about $10 \%$ of the 1997 sample defined as new statin users with a conventional definition of no statin purchases during the preceding year had purchased statins within the previous 3 years, the respective proportion in the 2007 sample was already about $20 \%$. In addition, the differences between those qualifying as new users with the 1-year gap but not with the 3-year gap seemed to be larger in 2007 than in 1997. One reason for the differences in the distribution of the initiating statin in 2007 may be the reimbursement restriction of atorvastatin and rosuvastatin [23]. In practice, the restriction meant that the initiators of atorvastatin and rosuvastatin with no trial with less expensive statins did not receive reimbursement for their statin and their purchases did not appear in the Prescription Register either. About half of those using atorvastatin 
and $40 \%$ of those using rosuvastatin in 2006 switched to simvastatin or another less expensive statin by the end of 2007 [23]. It is possible that not all statin purchases by the switchers were captured in the Prescription Register and some switchers may have appeared as new users in 2007. Therefore, the rates of misclassification with the shortest washout periods might be exceptionally high in 2007. Overall, our findings indicate that comparisons of incidence rates of statin use across time periods, population subgroups and between studies using different statin-free periods should be done cautiously.

Our observations on the differences in cohort characteristics according to the definition of new use may have implications especially for studies assessing acute health outcomes of statin use. For example, when an inception cohort of statin initiators in 2007 is defined based on 1 year versus 3 or more years of no statin purchases, the cohort is more likely to include older women due to their greater chance of being misclassified. Consequently, for example, the estimates of relative risks of potential adverse events such as rise in liver enzymes and myopathies with the peak in incidence during the first year of treatment [27] may be more biased in this than other population subgroups.

Our study has limitations. Despite their obvious generalizability to all ambulatory statin users in Finland and to those in other countries with similar health care and drug coverage systems, the results could be different, for example, in heterogenous North American populations with various prescription drug coverage plans [28]. Furthermore, we may have misclassified statin use, persistence and adherence for many reasons. First, we did not have data on hospitalizations nor is the drug use in hospital or other institutional care captured in the Register, potentially leading to overestimation of gaps in statin use and underestimation of adherence. Second, we assumed that the dosage for all users was 1 tablet per day. Because of splitting of tablets, we may have underestimated the adherence; however, splitting of tablets seems rare [29]. Conversely, prescribing of dosages of more than 1 tablet per day would have lead to overestimation of adherence and persistence. Finally, we approximated the patients' actual drug use based on dispensations, and therefore we could not be sure if and how the dispensed statins were used. Neither 
were we able to address the reasons why patients stopped or restarted their statin use $[15,30]$.

In conclusion, statin use is dynamic and reinitiations after treatment gaps are common. This should be taken into consideration in clinical practice and especially when studying the incidence, patterns and health outcomes of statin use.

\section{Aknowledgements}

This study was funded by a grant (October 26, 2007) from the Social Insurance Institution (SII) of Finland (Maarit Jaana Korhonen and Arja Helin-Samivaara).

We thank Kristiina Tyrkkö and Hilkka Ruuska of the SII for their expertise in extracting and managing the administrative data.

\section{References}

1. Baigent C, Keech A, Kearney PM, et al. (2005) Efficacy and safety of cholesterol-lowering treatment: prospective meta-analysis of data from 90,056 participants in 14 randomised trials of statins. Lancet. 366:1267-1278.

2. Brugts JJ, Yetgin T, Hoeks SE, et al. (2009) The benefits of statins in people without established cardiovascular disease but with cardiovascular risk factors: meta-analysis of randomised controlled trials. BMJ 338:b2376.

3. Shalev V, Chodick G, Silber H, et al. (2009) Continuation of statin treatment and all-cause mortality. Arch Intern Med 169:260-268. 
4. Perreault S, Dragomir A, Blais L, et al. (2009) Impact of better adherence to statin agents in the primary prevention of coronary artery disease. Eur J Clin Pharmacol 65:1013-1024.

5. Corrao G, Conti V, Merlino L, et al. (2010) Results of retrospective database analysis of adherence to statin therapy and risk of nonfatal ischemic heart disease in daily practice in Italy. Clin Ther 32:300-310.

6. Benner JS, Glyn RJ, Mogun H, et al. (2002) Long-term persistence in use of statin therapy in elderly patients. JAMA 288:455-461.

7. Mantel-Teeuwisse AK, Goettsh WG, Klungel OH, et al. (2004) Long term persistence with statin treatment in daily medical practice. Heart 90:1056-1066.

8. Perreault S, Blais L, Lamarre D, et al. (2005) Persistence and determinants of statin therapy among middle-aged patients for primary and secondary prevention. Br J Clin Pharmacol 59:564-573.

9. Helin-Salmivaara A, Lavikainen P, Korhonen MJ, et al. (2008) Long-term persistence with statin therapy: a nation-wide register study. Clin Ther 30(pt 2):2228-2240.

10. Helin-Salmivaara A, Lavikainen P, Korhonen MJ, et al. (2010) Pattern of statin use among 10 cohorts of new users from 1995 to 2004: A register-based nationwide study. Am J Manag Care 16:116-122.

11. Risselada R, Straatman H, van Kooten F, et al. (2009) Withdrawal of statins and risk of subarachnoid hemorrhage. Stroke 40:2887-2892.

12. Daskapoulou SS, Delaney JA, Fillion KB, et al. (2008) Discontinuation of statin therapy following an acute myocardial infarction: a population-based study. Eur Heart J 79:2083-2091. 
13. Pineda A, Cubeddu LX (2010) Statin rebound or withdrawal syndrome: Does it exist? Curr Athreoscler Rep DOI 10.1007/s11883-010-0148-x

14. Hudson M, Rahme E, Richard H, et al. (2007) Comparison of measures of medication persistency using a prescription drug database. Am Heart J 153:59-65.

15. Brookhart MA, Patrick AR, Schneeweiss S, et al. (2007) Physician follow-up and provider continuity are associated with long-term medication adherence. Arch Intern Med 167:847-852.

16. Ray WA (2003) Evaluating medication effects outside clinical trials: New-user designs. Am J Epidemiol 158:915-920.

17. Scranton RE, Young M, Lawler E, et al. (2005) Statin use and fracture risk. Arch Intern Med $165: 2007-2012$.

18. Willke RJ, Zhou S, Vogel RA (2008) Differences in cardiovascular event rates between atorvastatin and simvastatin among new users: managed-care experience. Curr Med Res Opin 24:2873-2882.

19. Smeeth L, Douglas I, Hall AJ, et al. (2008) Effect of statins on a wide range of health outcomes: a cohort study validated by comparison with randomized trials. Br J Clin Pharmacol 67: 99-109.

20. Chodick G, Heymann AD, Flash S, et al. (2010) Persistence with statins and incident cataract: a population-based historical cohort study. Ann Epidemiol 20:136-142.

21. Furu K, Wettermark B, Andersen M, et al. (2009) The Nordic countries as a cohort for 
pharmacoepidemiological research. Basic Clin Pharmacol Toxicol 106:86-94.

22. Peura S, Raijaniemi S, Kurkijärvi U. Pharmaceutical Pricing and Reimbursement Information Project. Finland 2007. Available at: http://ppri.oebig.at/Downloads/Results/Finland_PPRI_2007.pdf. Accessed Feb 1, 2010.

23. Martikainen JE, Saastamoinen LK, Korhonen MJ, et al. (2010) Impact of restricted reimbursement on the use of statins in Finland. A register-based study. Med Care 48:761-766.

24. WHO. About the ATC/DDD system. Available at: http://www.whocc.no/atcddd//. Accessed Feb 1, 2010.

25. Andrade SE, Kahler KH, Frech F, et al. (2006) Methods for evaluation of medication adherence and persistence using automated databases. Pharmacoepidemiol Drug Saf 15:565-574.

26. Simpson S, Eurich D, Majumdar S, et al. (2006) A meta-analysis of the association between adherence to drug therapy and mortality. BMJ 333(7557):15.

27. Hippisley-Cox J, Coupland C (2010) Unintended effects of statins in men and women in England and Wales: population based cohort study using the QResearch database. BMJ 340:c2197. doi:10.1136/bmj.c2197

28. Schneeweiss S, Patrick AR, Maclure M, et al. (2007) Adherence to statin therapy under drug cost sharing in patients with and without acute myocardial infarction: a population-based natural experiment. Circulation 115:2128-2135. 
29. Dormuth C, Scneeweiss S, Brookhart A, et al. (2007) Factors related to splitting tablets of HMG-CoA reductase inhibitors: A population based analysis in British Columbia. Pharmacoepidemiol Drug Saf 16:5137.

30. Steiner JF (2010) Can we identify clinical predictors of medication adherence . . . and should we? Med Care 48:193-195. 


\section{Figure legends}

Fig 1 Kaplan-Meier estimates of cumulative probability of reinitiating statin therapy in cohorts in which stopping therapy was defined as a tablet-free period of at least 90, 180 and 270 days after completion of a prescription. Index dates were the dates 90,180 and 270 days after the completion of the last prescription, respectively.

Fig 2 Proportions (\%) of persons maintaining good adherence and those with gaps of various lengths in good adherence for each year of follow-up. Only persons surviving until the end of each year included (see Table 2 for the numbers of patients in follow-up).

Fig 3 Proportions (\%) of persons misclassified as new users in 2007 when using statin-free periods of different lengths, by age and sex. Reference group: those with no statin dispensation during the preceding $\geq 10$ years. 
Table 1 Characteristics of statin initiators, defined using 1- and 3-year gaps of no statin purchases before the first purchase in 1997 and 2007

\begin{tabular}{lcccc}
\hline & \multicolumn{2}{c}{1997} & \multicolumn{2}{c}{2007} \\
\cline { 2 - 5 } Characteristic & 3-year gap & 1-year gap & 3-year gap & 1-year gap \\
\hline No. of persons & 32760 & 36581 & 78512 & 99348 \\
Male, n (\%) & $15534(47.4)$ & $17653(48.3)$ & $41891(53.4)$ & $52142(52.5)$ \\
Age, mean (SD), y & $63.0(8.9)$ & $63.0(8.9)$ & $62.5(9.6)$ & $62.9(9.7)$ \\
Age, n (\%) & & & \\
45-54 y & $6685(20.4)$ & $7440(20.3)$ & $17635(22.5)$ & $21156(21.3)$ \\
$55-64$ y & $10937(33.4)$ & $12310(33.7)$ & $30368(38.7)$ & $37876(38.1)$ \\
$65-74$ y & $11983(36.6)$ & $13317(36.4)$ & $19499(24.8)$ & $25420(25.6)$ \\
$75-84$ y & $3155(9.6)$ & $3514(9.6)$ & $11010(14.0)$ & $14896(15.0)$ \\
Initial statin, n (\%) & & & & \\
Simvastatin & $14952(45.6)$ & $16513(45.1)$ & $70123(89.3)$ & $84787(85.3)$ \\
Fluvastatin & $10362(31.6)$ & $11268(30.8)$ & $2170(2.8)$ & $3364(3.4)$ \\
Lovastatin & $5372(16.4)$ & $6478(17.7)$ & $299(0.4)$ & $724(0.7)$ \\
Pravastatin & $2074(6.3)$ & $2322(6.4)$ & $987(1.3)$ & $1832(1.8)$ \\
Atorvastatin & NA & NA & $2750(3.5)$ & $5032(5.1)$ \\
Rosuvastatin & NA & NA & $1661(2.1)$ & $2833(2.9)$ \\
Simvastatin+Ezetimibe & NA & NA & $522(0.7)$ & $776(0.8)$ \\
\hline Stan initiar
\end{tabular}

${ }^{a}$ Statin initiator defined as a person with no statin purchases within the preceding 1095 days and

${ }^{\mathrm{b}}$ as a person with no statin purchases within the preceding 365 days. 
Table 2 Mean, median and interquartile range of the proportion of day covered (PDC) and proportions of those with good adherence (PDC $\geq 0.80$ ) for each of the 10 years of follow-up in the 1997 cohort of statin initiators.

\begin{tabular}{|c|c|c|c|c|c|}
\hline \multirow{3}{*}{$\begin{array}{l}\text { Year Since } \\
\text { Initiation }\end{array}$} & \multicolumn{5}{|c|}{ Proportion of Days Covered } \\
\hline & No. of & & & Interquartile & Good \\
\hline & Persons ${ }^{a}$ & Mean & Median & Range & Adherence (\%) \\
\hline 1 & 30893 & 0.67 & 0.78 & $0.45-0.93$ & 48.2 \\
\hline 2 & 29489 & 0.58 & 0.72 & $0.25-0.92$ & 43.6 \\
\hline 3 & 28156 & 0.60 & 0.75 & $0.25-0.93$ & 46.1 \\
\hline 4 & 26930 & 0.62 & 0.80 & $0.27-0.94$ & 49.8 \\
\hline 5 & 25789 & 0.64 & 0.82 & $0.33-0.95$ & 52.1 \\
\hline 6 & 24655 & 0.65 & 0.83 & $0.38-0.95$ & 53.6 \\
\hline 7 & 23530 & 0.67 & 0.85 & $0.44-0.96$ & 55.7 \\
\hline 8 & 22353 & 0.68 & 0.87 & $0.49-0.96$ & 58.2 \\
\hline 9 & 21155 & 0.69 & 0.87 & $0.52-0.96$ & 58.6 \\
\hline 10 & 20064 & 0.70 & 0.87 & $0.53-0.96$ & 58.8 \\
\hline
\end{tabular}

${ }^{a}$ Persons surviving until the end of the year 


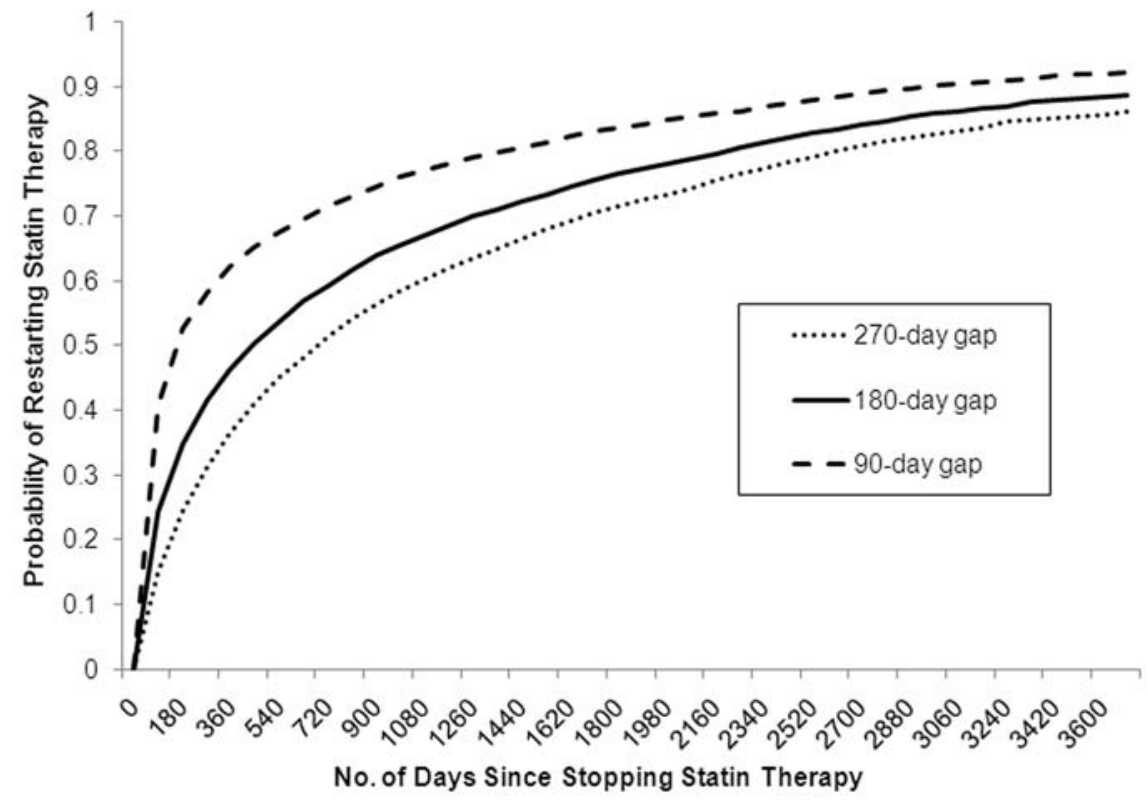




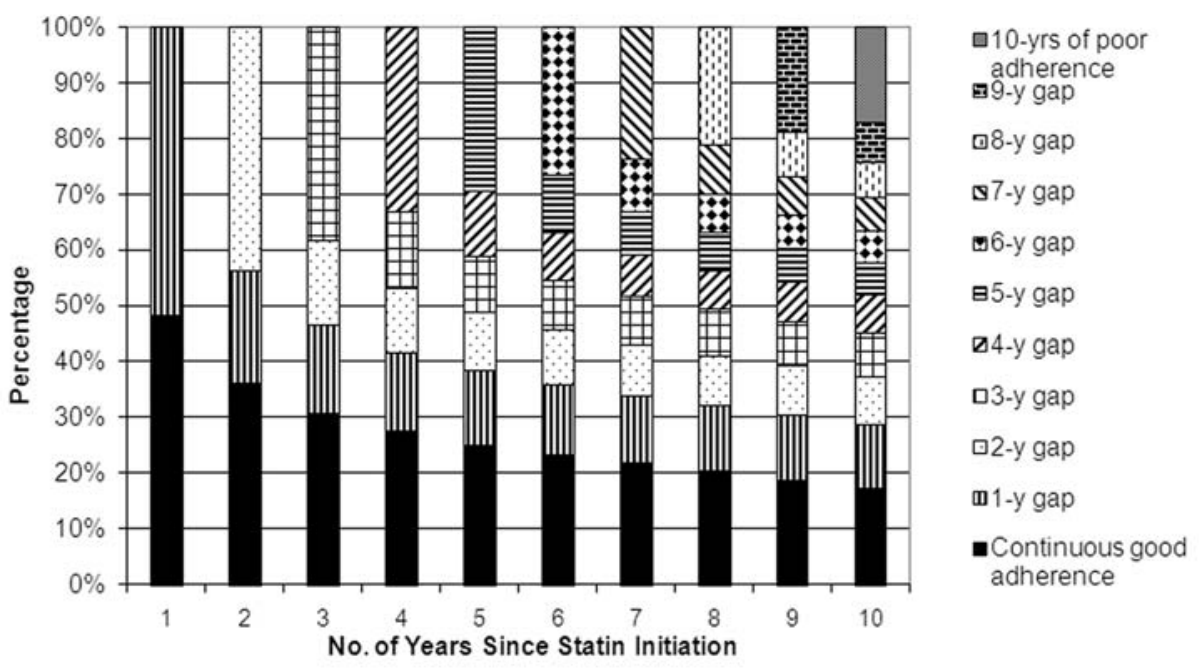




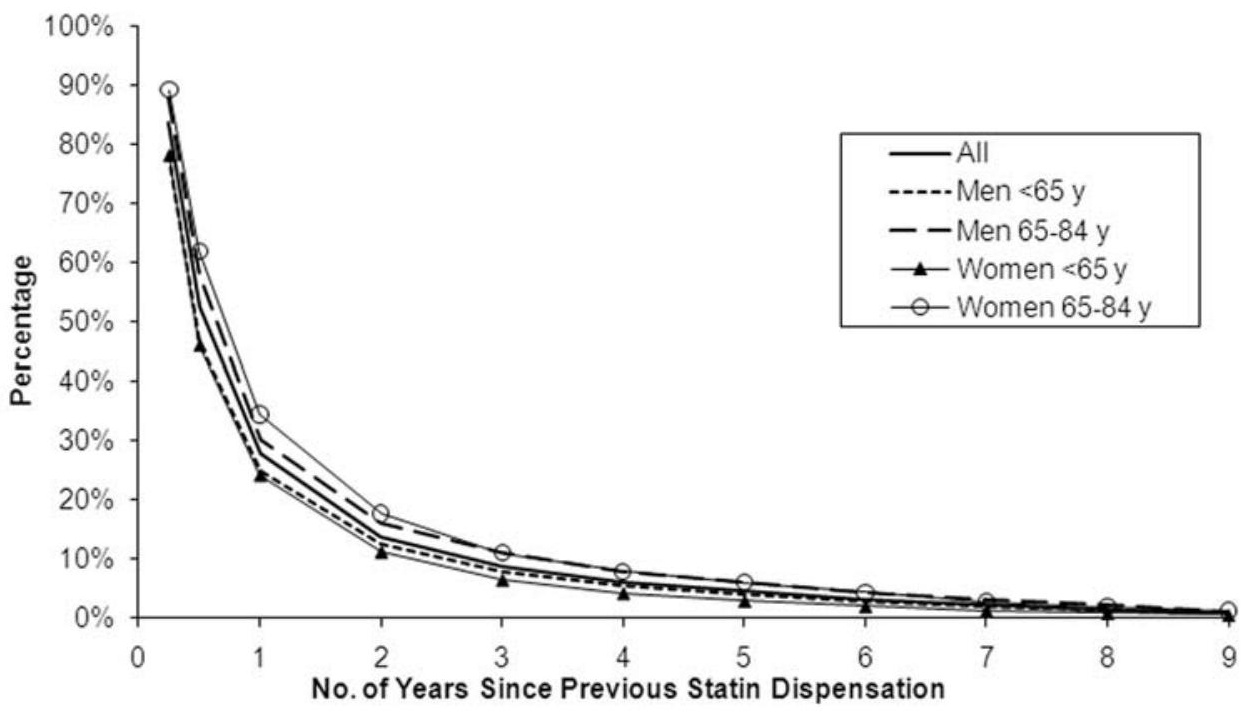

